\title{
Assessment of Photon Recycling in Perovskite Solar Cells by Fully Coupled Optoelectronic Simulation
}

\author{
Simon Zeder@, ${ }^{*}$ Beat Ruhstaller $\odot,^{\dagger}$ and Urs Aeberhard $\odot^{\dagger}$ \\ Fluxim AG, Winterthur 8400, Switzerland
}

(Received 2 September 2021; revised 8 November 2021; accepted 8 December 2021; published 20 January 2022)

\begin{abstract}
An optical dyadic Green's function framework to describe the transverse electromagnetic fields in a planar perovskite solar-cell stack is coupled to an electronic drift-diffusion model for rigorous treatment of photon recycling in the wave-optics regime for a realistic photovoltaic device. The optical model provides the local reabsorption rate as well as a detailed-balance compatible radiative prefactor, which are used in the electronic model to achieve a self-consistent solution that yields the full optoelectronic device characteristics. The presented approach provides detailed insights into the impact of photon recycling on device performance under different regimes of charge transport and recombination and can help identify the various electronic and optical losses for nonideal, realistic devices. The global efficiency of photon recycling is quantified by defining quantum efficiencies of reabsorbed radiation, while the local efficiency can furthermore be quantified by defining an effective local radiative prefactor. The model introduced here can be used to guide the design of future devices that exploit the full potential of photon recycling.
\end{abstract}

DOI: 10.1103/PhysRevApplied.17.014023

\section{INTRODUCTION}

In the past few years, hybrid metal-halide perovskite materials have become more and more popular for use in single-junction and multijunction (tandem) solar cells, especially in combination with crystalline silicon, where for both device architectures new record efficiencies could be reached recently ( $>25 \%$ for single-junction [1] and $>29 \%$ for Si tandem cells [2]). Similarly, perovskite semiconductors have been gaining attention also for use in light-emitting devices. One aspect making this material ideally suited for photovoltaic energy conversion is the strong optical absorption and remarkably low nonradiative recombination for high-quality perovskites, which allows such devices to operate close to the radiative limit [3-5]. Furthermore, an exceptionally large joint density of

\footnotetext{
*simon.zeder@fluxim.com

Also at École Polytechnique Fédérale de Lausanne, PV-LAB, Institute of Microengineering, Neuchâtel 2002, Switzerland.

†beat.ruhstaller@fluxim.com

Also at Zurich University of Applied Science, Institute of Computational Physics, Winterthur 8401, Switzerland.

†urs.aeberhard@fluxim.com

Also at Eidgenössische Technische Hochschule Zürich, Integrated Systems Laboratory, Zürich 8092, Switzerland.

Published by the American Physical Society under the terms of the Creative Commons Attribution 4.0 International license. Further distribution of this work must maintain attribution to the author(s) and the published article's title, journal citation, and DOI.
}

states (JDOS) close to the band edge leads to a very sharp absorption edge in these materials [6], which in turn results in a low Stokes shift and hence a pronounced overlap of absorption and emission spectra. The combination of these properties leads to sizable photon-recycling (PR) effects due to reabsorption of internally emitted photons $[7,8]$ with a positive effect on device performance, such as an increase in open-circuit voltage $\left(V_{\mathrm{OC}}\right)$ [9] for photovoltaic devices and improved external quantum efficiency due to the reabsorption and re-emission of guided into leaky modes for light-emitting devices [10]. Modeling and simulation can play a decisive role in understanding these processes as internal emission and reabsorption are difficult to assess experimentally.

Modeling of PR coupled to electronic transport has already been employed for some time, most prominently in GaAs (gallium arsenide) photovoltaic devices, however, largely based on ray-optical approaches [11-13]. Treatments of PR beyond the ray-optical approximation in thin-film perovskite devices have been limited to purely optical estimates of the open-circuit voltage enhancement using either a detailed-balance approach $[9,14,15]$ or a rigorous solution of Maxwell's equations in the dipole picture $[16,17]$ for external and internal emission. While the former usually relies on the generalized Kirchhoff law [18] for external emission, which correctly takes the optical environment of the full device into account, and on the van Roosbroeck-Shockley (VRS) relation [19] for internal emission, which assumes emission into an optically homogeneous medium (and which is therefore inconsistent 
with the aforementioned generalized Kirchhoff law), the latter calculates the internal emission taking the wave optics of the device into account. Yet, these dipole models suffer, on the one hand, from divergencies in emitted power due to nonradiative coupling to evanescent modes in the absorbing surrounding medium [20-22], which has to be circumvented by introducing nonphysical transparent regions around the dipole [16,23], and, on the other hand, from the lack of a detailed-balance compatible connection to the electronic excitation giving rise to the emission in the first place. In general, the full coupling of a wave-optical treatment of emission and reabsorption to the electronic transport problem beyond the radiative limit in a detailed-balance compatible way has not been achieved so far, as would be needed for a correct understanding of this phenomenon in thin-film perovskite solar cells. Such understanding would enable the further optimization of solar-cell designs to increase PR, the study of which has seen an increased interest recently (see, e.g., Raja et al. [24] and Cheng and O'Carroll [25] for recent reviews).

In this paper we present such a rigorous and fully coupled approach and apply it to a model system of a high- $V_{\mathrm{OC}}$ device from the literature, in order to analyze the mechanisms and benefits of PR in realistic optical and electronic conditions (multilayer thin-film device, parasitic absorption, nonradiative recombination, nonideal band alignment, ...). The first part of the paper gives a short overview of the employed optical and electronic model and the coupling thereof. Then the description of the photovoltaic devices studied is given followed by a quantification of the benefits of PR, an analysis of the electrical losses and finally an assessment of the optical losses, both losses resulting in a deviation of the ideally achievable $V_{\mathrm{OC}}$ enhancement due to PR. The paper is wrapped up with a summary and conclusion of the findings.

\section{COMPUTATIONAL MODEL}

In order to describe the physical processes in the solar cell, a mathematical model consisting of two parts is employed. On the one hand, the optical characteristics of the layered system are described using a dyadic Green's function approach, which depends only on the stack geometry and the optical constants (complex refractive indices) of the materials. On the other hand, electronic transport processes are described using an established drift-diffusion model capable of simulating complex multilayer device architectures and processes including nonradiative recombination.

For sake of space and clarity, only the crucial parts of the optical model are described here, with the fully detailed description given in Ref. [26]. For a quasi-onedimensional (1D) system where the in-plane dimensions are assumed to be infinitely extended the problem assumes an in-plane rotational symmetry such that it is beneficial to use cylindrical coordinates $(\vec{\varrho}, z)$ with $\vec{\varrho}$ the in-plane vector $\vec{\varrho}=\vec{r}_{\|}-\vec{r}_{\|}^{\prime}$. Furthermore, the real-space dyadic Green's tensor $\stackrel{\leftrightarrow}{G}\left(\vec{\varrho}, z, z^{\prime}, E_{\gamma}\right)$ is preferably expressed in terms of its partial Fourier transform

$$
\begin{aligned}
\stackrel{\leftrightarrow}{G}\left(\mathbf{r}, \mathbf{r}^{\prime}, E_{\gamma}\right) & \equiv \stackrel{\leftrightarrow}{G}\left(\vec{\varrho}, z, z^{\prime}, E_{\gamma}\right) \\
& =\int \frac{d^{2} \vec{q}_{\|}}{(2 \pi)^{2}} \stackrel{\leftrightarrow}{G}\left(\vec{q}_{\|}, z, z^{\prime}, E_{\gamma}\right) \exp \left(\overrightarrow{i q}_{\|} \cdot \vec{\varrho}\right),
\end{aligned}
$$

where $E_{\gamma}$ is the photon energy and $\vec{q}_{\|}$the in-plane photon momentum. For sources $z^{\prime}$ lying inside or in proximity to absorbing layers, the integral in Eq. (1) diverges due to the nonradiative coupling to longitudinal (evanescent) photonic modes [20-22]. This can be circumvented by realizing that for inorganic, nonexcitonic semiconductors radiative recombination primarily couples to transverse photons, which are related to the transverse part of the Green's tensor $\stackrel{\leftrightarrow}{G}_{T}\left(\mathbf{r}, \mathbf{r}^{\prime}, E_{\gamma}\right)$ [21,27-29], which is obtained here by subtracting the purely longitudinal part from the total Green's tensor. For simplicity, the subscript ${ }_{T}$ is omitted and $\stackrel{\leftrightarrow}{G}$ from now on directly refers to the transverse Green's tensor.

Once the transverse Green's tensor is computed for the given optical layer stack, it can be used to calculate the necessary input quantities for the electronic transport simulation. First, a detailed-balance compatible radiative recombination rate is obtained based on a general nonequilibrium quantum-kinetic framework (NEGF) [30] (and consistent with Würfel's general formulations [18])

$$
\begin{aligned}
\mathcal{R}_{\mathrm{rad}}(z)= & \int d E_{\gamma} \alpha\left(z, E_{\gamma}\right) \mathcal{D}_{\gamma}\left(z, E_{\gamma}\right) \frac{c_{0}}{n_{r}\left(z, E_{\gamma}\right)} \\
& \times f_{\mathrm{BE}}\left[E_{\gamma}-\Delta \mu_{c v}(z)\right] \\
\approx & \int d E_{\gamma} \frac{4 E_{\gamma}^{2}}{\pi \hbar^{3} c_{0}^{2}} n_{r}\left(z, E_{\gamma}\right) \kappa\left(z, E_{\gamma}\right) \\
& \times \operatorname{Im}\left[\operatorname{Tr} \stackrel{\leftrightarrow}{G}\left(z, z, E_{\gamma}\right)\right] \\
& \times \exp \left(-\frac{E_{\gamma}}{k_{B} T}\right)\left[\frac{n(z) p(z)}{n_{i}^{2}(z)}-1\right] \\
\equiv & \mathcal{B}_{\mathrm{rad}}(z)\left[n(z) p(z)-n_{i}^{2}(z)\right]
\end{aligned}
$$

where $\alpha, \mathcal{D}_{\gamma}, n_{r}, \kappa$, and $\Delta \mu_{c v}$ are the absorption coefficient, the local photonic density of states (LDOS), real and complex part of the refractive index, and quasi-Fermilevel splitting, respectively. In the second expression we calculate the net emission rate by expressing the LDOS in terms of the imaginary part of the transverse Green's tensor and employing the Boltzmann approximation to express the quasi-Fermi-level splitting in terms of the electron and hole densities ( $n$ and $p$, respectively) and the squared intrinsic charge density $n_{i}^{2}$. 
Furthermore, it is possible to derive an expression describing the local reabsorption rate caused by radiative emission at all other points $z^{\prime}$ in the layer stack:

$$
\begin{aligned}
\mathcal{G}_{\text {reabs }}(z)= & \int d E_{\gamma} \frac{8 E_{\gamma}^{4}}{\pi \hbar^{5} c_{0}^{4}} n_{r}\left(z, E_{\gamma}\right) \kappa\left(z, E_{\gamma}\right) \\
& \times \int d z^{\prime} n_{r}\left(z^{\prime}, E_{\gamma}\right) \kappa\left(z^{\prime}, E_{\gamma}\right) \exp \left(-\frac{E_{\gamma}}{k_{B} T}\right) \\
& \times\left[\frac{n\left(z^{\prime}\right) p\left(z^{\prime}\right)}{n_{i}^{2}\left(z^{\prime}\right)}-1\right] \\
& \times \sum_{\mu, \nu} \int \frac{d^{2} \vec{q}_{\|}}{(2 \pi)^{2}}\left|G_{\mu \nu}\left(\vec{q}_{\|}, z, z^{\prime}, E_{\gamma}\right)\right|^{2} .
\end{aligned}
$$

It has been shown that such a local approach is fully compatible with global detailed-balance relations such as the generalized Kirchhoff relation between global quasi-Fermi-level splitting and emitted photoluminescence spectra $[18,26]$, which are frequently used for the characterization of solar cells [3,31,32].

Going beyond the analysis in Ref. [26], the optical model is now coupled to an electronic model to include realistic transport aspects. To this end, the two quantities from Eqs. (2) and (3) are included directly in the driftdiffusion formulation of the electronic transport problem, consisting of the Poisson equation and the electron and hole continuity equations, as implemented in the optoelectronic device simulator SETFOS [33]

$$
\begin{aligned}
& \frac{\partial}{\partial z}\left[\varepsilon(z) \frac{\partial}{\partial z} \psi(z, t)\right]=q(n(z, t)-p(z, t)+\ldots), \\
& \frac{\partial n}{\partial t}=\frac{\partial}{\partial z} J_{n}(z, t)-\mathcal{R}(z, t)+\mathcal{G}(z, t), \\
& \frac{\partial p}{\partial t}=\frac{\partial}{\partial z} J_{p}(z, t)-\mathcal{R}(z, t)+\mathcal{G}(z, t),
\end{aligned}
$$

where $\psi$ is the electrostatic potential (with the right-hand side of Eq. (4a) representing the sum of all charges present in the system), $J_{n}$ and $J_{p}$ the electron and hole current densities, respectively, and

$$
\begin{aligned}
\mathcal{R}(z, t) & =\mathcal{B}_{\text {rad }}(z)\left[n(z, t) p(z, t)-n_{i}^{2}(z)\right]+\mathcal{R}_{\mathrm{SRH}}(z, t), \\
\mathcal{G}(z, t) & =\mathcal{G}_{\text {illum }}(z, t)+\mathcal{G}_{\text {reabs }}(z, t),
\end{aligned}
$$

with $\mathcal{R}_{\mathrm{SRH}}$ given by the usual expression for trap-assisted Shockley-Read-Hall (SRH) recombination [34]. The radiative rate prefactor $\mathcal{B}_{\text {rad }}$ and the reabsorption rate $\mathcal{G}_{\text {reabs }}$ are computed from the optical model based on the Green's function. $\mathcal{G}_{\text {illum }}$ represents the charge generation caused by the external illumination and is calculated using the transfer-matrix method available in SETFOS [33]. To ensure consistency and detailed-balance compatibility (discussed

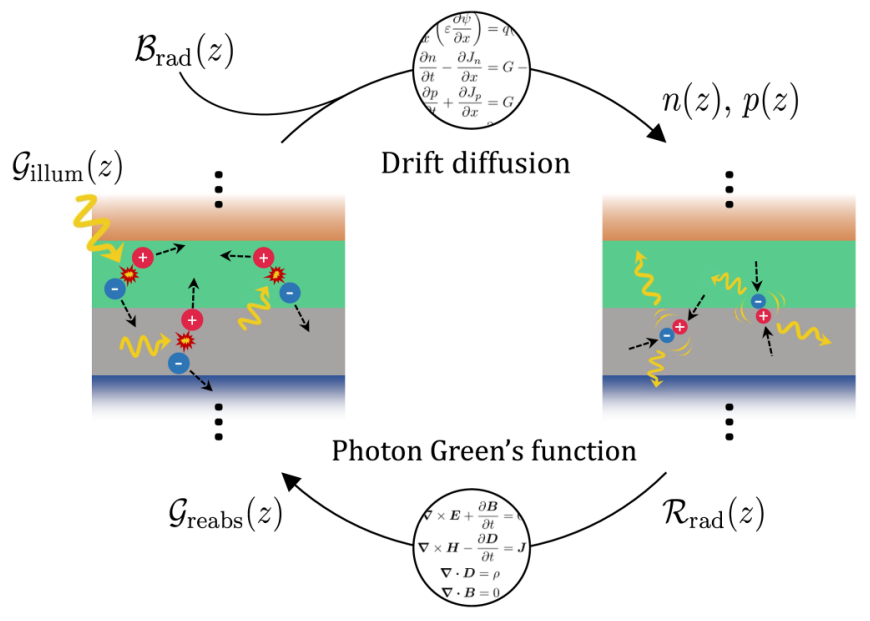

FIG. 1. Iterative PR scheme coupling optical and electronic transport models.

in Ref. [26]), the same complex refractive index data was used for the computation of all optoelectronic quantities $\left(\mathcal{B}_{\text {rad }}, \mathcal{G}_{\text {reabs }}\right.$, and $\left.\mathcal{G}_{\text {illum }}\right)$. The computation of $\mathcal{G}_{\text {reabs }}$ depends on the unknown electron and hole densities $n$ and $p$ through radiative recombination, hence an iterative approach is required. In this approach, once the Green's functions for the given layer stack are calculated, the local generation rate due to $\mathrm{PR}$ is updated after each solution of $n$ and $p$ using the aforementioned Green's function model, which again in turn gives rise to an updated $n$ and $p$ distribution (shown schematically in Fig. 1).

\section{RESULTS AND DISCUSSION}

The optoelectronic model introduced above can now be applied to a realistic solar-cell-device structure. As model systems the two high- $V_{\mathrm{OC}}$ device architectures presented by Liu et al. [32] are implemented, which both consist of an indium tin oxide (ITO) transparent electrode $(150 \mathrm{~nm})$, polytriarylamine (PTAA) as hole transport layer (HTL, 30/12 nm), a methylammonium lead iodide (MAPI) perovskite absorber layer $(280 / 510 \mathrm{~nm})$ and phenyl-C61butyric acid methyl ester (PCBM) as electron transport layer (ETL, $45 \mathrm{~nm}$ ). The bottom electrode consists of a thin bathocuproine $(\mathrm{BCP})$ layer $(8 \mathrm{~nm})$ and a silver $(\mathrm{Ag})$ back reflector $(80 \mathrm{~nm})$. Both layer stacks are displayed schematically in Figs. 2(a) and 2(b). A fit of the measured $J-V$ characteristics is then performed using the SETFOS device simulation software [33] by varying the SRH (trap) parameters as well as the electron and hole mobilities in the MAPI, HTL, and ETL. The fitted $J-V$ curves along with the measured characteristics are shown in Fig. 2(c) for both architectures.

The fit is performed with the additional generation due to PR being taken into account, as PR is assumed to be present also in the actual measurement. It is assumed that 

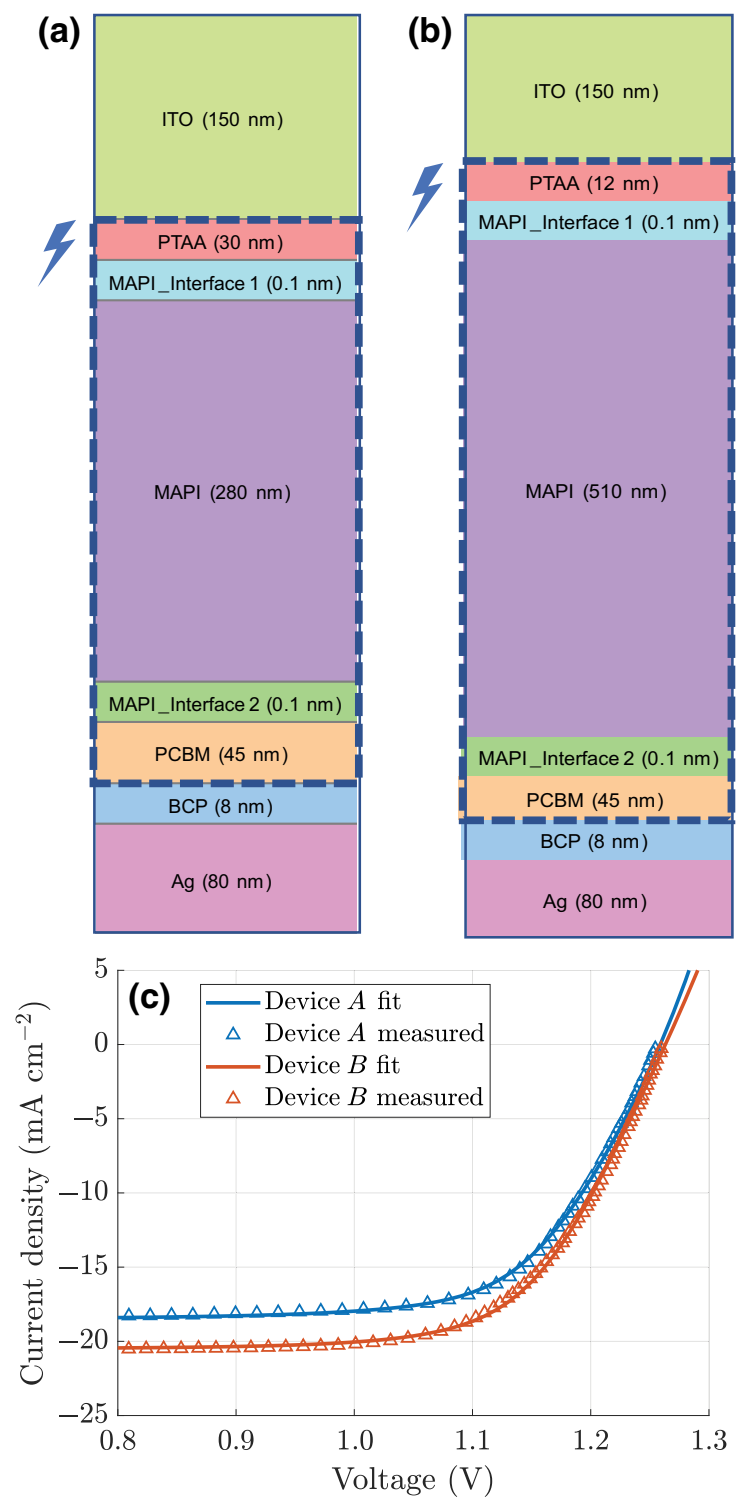

FIG. 2. Layer stack structures for the two devices under consideration (the substack considered in the electronic transport simulation is marked with the blue dashed line). (a) Device $A$ geometry. (b) Device $B$ geometry. (c) Measured and fitted $J-V$ characteristics for both devices close to $V_{\mathrm{OC}}$.

radiative generation and recombination is restricted to the MAPI absorber. On the other hand, trapping with SRH recombination is present throughout the entire electrically active stack where additional thin $(0.1 \mathrm{~nm})$ interface layers are included at the PTAA-MAPI and MAPI-PCBM interfaces with the same electrical characteristics as MAPI but with increased SRH recombination rates in order to model strong recombination. The values of the parameters chosen for the drift-diffusion simulation are given within the Supplemental Material [35] and compared to the literature [32,33,36-44], alongside the complex refractive-index data used in the optical models [32,45-49].

\section{A. Electrical-loss analysis}

It is well known that PR in GaAs and perovskite solar cells (among others) leads to an increased $V_{\mathrm{OC}}$ due to an enhanced quasi-Fermi-level splitting in the active region. Often this is modeled using an effective (reduced) prefactor for the radiative recombination rate [14,50], which however lacks any spatial information. Furthermore, the effectiveness of PR strongly depends on the nonradiative recombination channels, which can quickly quench any benefit from PR if dominant [51]. By employing a local approach as presented here such issues can be taken into account directly.

A comparison of the $J-V$ characteristics of the two devices in the presence or absence of PR is shown in Figs. 3(a) and 3(b), respectively. The open-circuit voltage and power-conversion-efficiency (PCE) enhancement is shown in Fig. 3(c) for the case where nonradiative SRH recombination is taken into account, in the radiative limit (i.e., zero nonradiative recombination) as well as in a purely optical limit (neglecting any electronic transport losses and assuming a spatially constant quasiFermi-level splitting corresponding to the applied voltage). The enhancement in $V_{\mathrm{OC}}$ and PCE is obviously smaller in the realistic case compared to the radiative limit, as the nonradiative recombination quenches the internal emission and hence reduces the extent of possible PR (see also Fig. S4 within the Supplemental Material [35]). The optical limit is computed using a current-voltage characteristic of an ideal diode:

$$
J(V)=-J_{\mathrm{SC}}+J_{0, \mathrm{rad}}\left[\exp \left(\frac{q V}{k_{B} T}\right)-1\right],
$$

with $J_{\mathrm{SC}}$ the optical short-circuit current and $J_{0, \text { rad }}$ the radiative (ideal) dark saturation current. While $J_{\mathrm{SC}}$ is pretty much equal in all cases for a given device, the dark saturation current has to be calculated from the radiative recombination current in the active absorber

$$
\begin{aligned}
J_{0, \mathrm{rad}}^{\dagger}= & \frac{4 q}{\pi \hbar^{3} c_{0}^{2}} \int d E_{\gamma} E_{\gamma}^{2} \exp \left(-\frac{E_{\gamma}}{k_{B} T}\right) \\
& \times \int d z n_{r}\left(z, E_{\gamma}\right) \kappa\left(z, E_{\gamma}\right) \operatorname{Im}\left[\operatorname{Tr} \stackrel{\leftrightarrow}{G}\left(z, z, E_{\gamma}\right)\right] \\
J_{0, \mathrm{rad}}= & J_{0, \mathrm{rad}}^{\dagger}-\frac{8 q}{\pi \hbar^{5} c_{0}^{4}} \int d E_{\gamma} E_{\gamma}^{4} \exp \left(-\frac{E_{\gamma}}{k_{B} T}\right) \\
& \times \int d z n_{r}\left(z, E_{\gamma}\right) \kappa\left(z, E_{\gamma}\right) \int d z^{\prime} n_{r}\left(z^{\prime}, E_{\gamma}\right) \kappa\left(z^{\prime}, E_{\gamma}\right) \\
& \times \sum_{\mu, \nu} \int \frac{d^{2} \vec{q}_{\|}}{(2 \pi)^{2}}\left|G_{\mu \nu}\left(\vec{q}_{\|}, z, z^{\prime}, E_{\gamma}\right)\right|^{2},
\end{aligned}
$$

with the dagger representing the case without reabsorption taken into account. $J_{0, \text { rad }}^{\dagger}$ in Eq. (6) represents the 

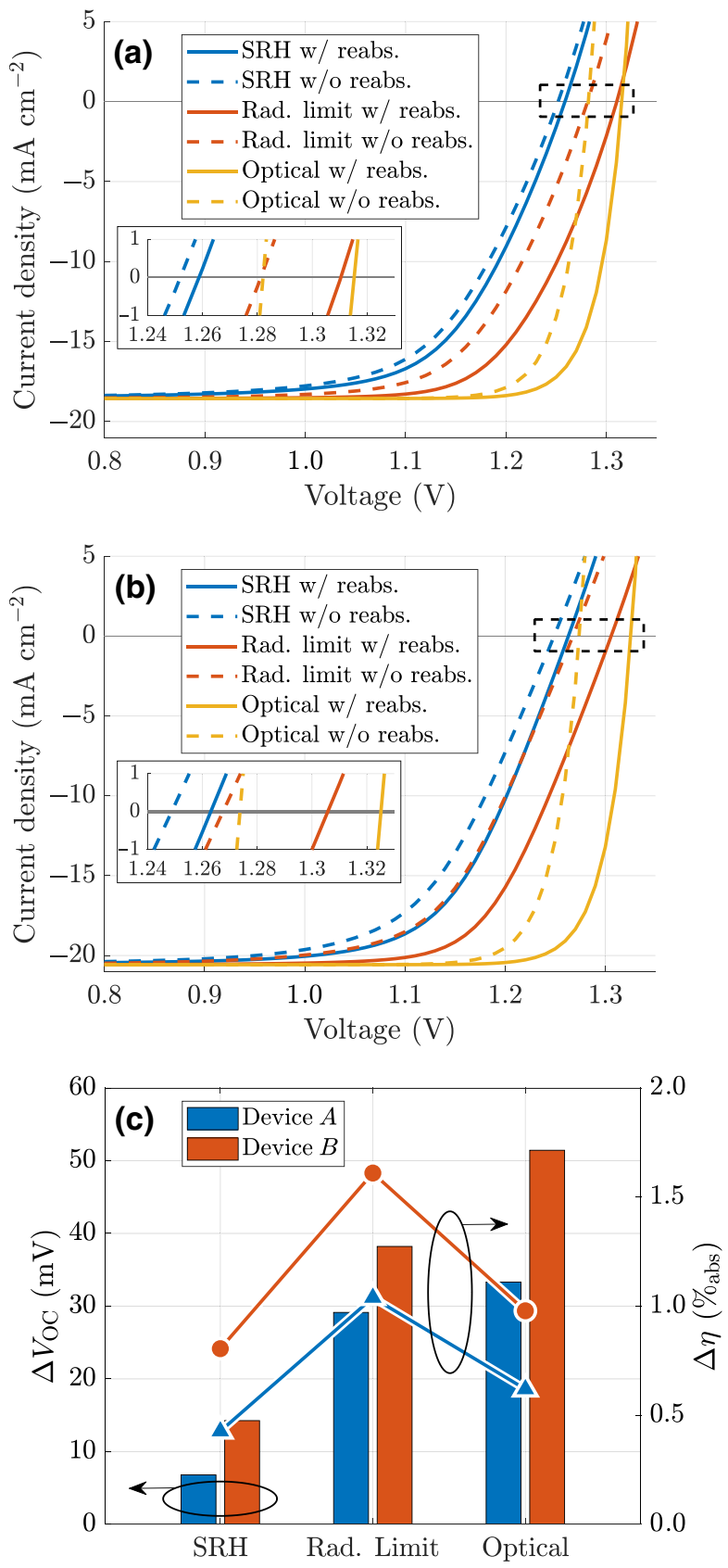

FIG. 3. Comparison of electrical key figures of both devices with and without PR. (a) $J-V$ characteristics for three levels of ideality for device $A$. (b) Same for device $B$. The inset shows a detailed view of the marked area around $V_{\mathrm{OC}}$. (c) Enhancement of $V_{\mathrm{OC}}$ (bars) and PCE (lines) due to PR with SRH recombination, in the radiative limit and optical limit. See also Fig. S4 and corresponding discussion within the Supplemental Material [35].

energy-integrated local radiative emission, while the second term in Eq. (7) corrects this value by the amount that is reabsorbed in the MAPI layer. Employing such an ideal diode model, Eq. (5), implicitly assumes that the applied voltage directly governs the quasi-Fermi-level splitting in the active absorber and therefore any voltage loss in the charge-transport layers (due to, e.g., band misalignment, internal resistance due to finite mobilities, ...) is neglected. The values calculated by this method hence represent absolute upper limits for a given optical device structure.

While device $B$ still shows a considerable discrepancy between the radiative and the optical limit and hence room for improvement when it comes to $(\Delta) V_{\mathrm{OC}}$, device $A$ gets already very close to this absolute performance limit. The fill factor for both devices under consideration of transport is, however, still far from ideal, which can be explained by reduced extraction efficiency and finite mobilities when taking the full device into account.

For a more detailed analysis of the device behavior we focus on device $B$, as both architectures exhibit similar behavior in this regard and a conclusion based on the characteristics of one also holds for the other device. The plots showing the data for device $A$ can be found within the Supplemental Material [35]. Only radiative recombination in the bulk MAPI and SRH recombination in the interface layers are considered, as other contributions are negligible in comparison.

The radiative recombination rate in the MAPI is governed by the radiative rate prefactor $\mathcal{B}_{\text {rad }}$, which is calculated from Eq. (2). By taking the photonic environment (LDOS) into account, considerable deviations from the VRS approximation arise. The VRS expression is based on detailed-balance considerations, however, assuming emission into an optically homogeneous medium, and is given by [19]

$$
\begin{aligned}
\mathcal{B}_{\mathrm{rad}}^{\mathrm{VRS}}(z)= & \frac{2}{\pi^{2} \hbar^{4} c^{3} n_{i}^{2}(z)} \int d E_{\gamma} E_{\gamma}^{3} n_{r}^{2}\left(z, E_{\gamma}\right) \kappa\left(z, E_{\gamma}\right) \\
& \times \exp \left(-\frac{E_{\gamma}}{k_{B} T}\right)
\end{aligned}
$$

In Fig. 4 the radiative prefactor in the MAPI layer of device $B$ is plotted, calculated using the VRS expression from Eq. (8) (solid line) and using our Green-function-based expression from Eq. (2). As the complex refractive index is constant in the MAPI layer, the radiative prefactor in the VRS approximation $\mathcal{B}_{\text {rad }}^{\mathrm{VRS}}$ is also constant over the layer thickness, whereas the LDOS introduces significant spatial variation of $\mathcal{B}_{\text {rad }}$ as calculated from the Green's function.

As shown in Fig. 5(a), the spatially integrated recombination rates due to radiative transitions and trap-assisted SRH processes are of similar magnitude with some variation depending on the external voltage. As mentioned, the radiative recombination occurs mainly in the bulk MAPI absorber while the nonradiative recombination is split between the two interfaces with the CTLs, with the interface to the HTL tending to be dominant depending on the external voltage. This picture might be misleading, however, as it shows the gross radiative recombination 


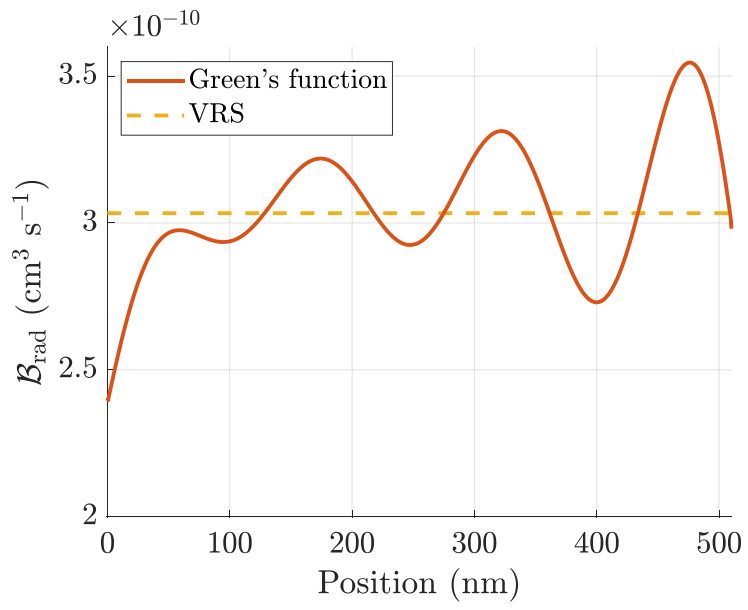

FIG. 4. Comparison of the radiative rate prefactor $\mathcal{B}_{\text {rad }}$ in the MAPI layer of device $B$ as calculated by the van RoosbroeckShockley (VRS) expression and using our Green's function model.

rate as calculated from Eq. (2). In order to quantify the actual losses in the system it is more appropriate to use an effective radiative recombination rate defined as

$$
\mathcal{R}_{\mathrm{rad}}^{\mathrm{eff}}(z) \equiv \mathcal{R}_{\mathrm{rad}}(z)-\mathcal{G}_{\text {reabs }}(z),
$$

which describes the local net loss due to radiative recombination. If this effective radiative recombination rate is considered, the relative loss balance shifts more towards nonradiative losses as shown in Fig. 5(b). Even though at first glance this seems like a change for the worse, a strong relative decrease in radiative recombination is a direct effect of efficient PR. Close to short-circuit conditions, $\mathcal{R}_{\text {rad }}^{\text {eff }} \approx \mathcal{R}_{\text {rad }}$ as PR is negligible there. The profiles of the absolute values of $\mathcal{R}_{\text {rad }}(z)$ and $\mathcal{G}_{\text {reabs }}(z)$ for both devices are plotted in Fig. S7 within the Supplemental Material [35].

While the enhancement of the open-circuit voltage provides a direct measure of the absolute magnitude of PR in a given device, a useful quantity for the assessment and optimization of the PR efficiency can be defined in the shape of a reabsorption internal/external quantum efficiency (IQE/EQE) as follows:

$$
\begin{gathered}
\eta_{\mathrm{IQE}}^{\text {reabs }}(V) \equiv \frac{J_{\dot{\dagger}}^{\mathrm{inj}}(V)-J^{\mathrm{inj}}(V)}{q}\left[\int d z \mathcal{G}_{\text {reabs }}(z, V)\right]^{-1}, \\
\eta_{\mathrm{EQE}}^{\text {reabs }}(V) \equiv \frac{J_{\dot{\dagger}}^{\mathrm{inj}}(V)-J^{\mathrm{inj}}(V)}{q}\left[\int d z \mathcal{R}_{\mathrm{rad}}(z, V)\right]^{-1},
\end{gathered}
$$

where $J^{\text {inj }}$ and $J_{\dot{\dagger}}^{\text {inj }}$ represent the injected current densities at the terminals with and without PR, respectively. The quantity in Eq. (10) describes the collection efficiency of
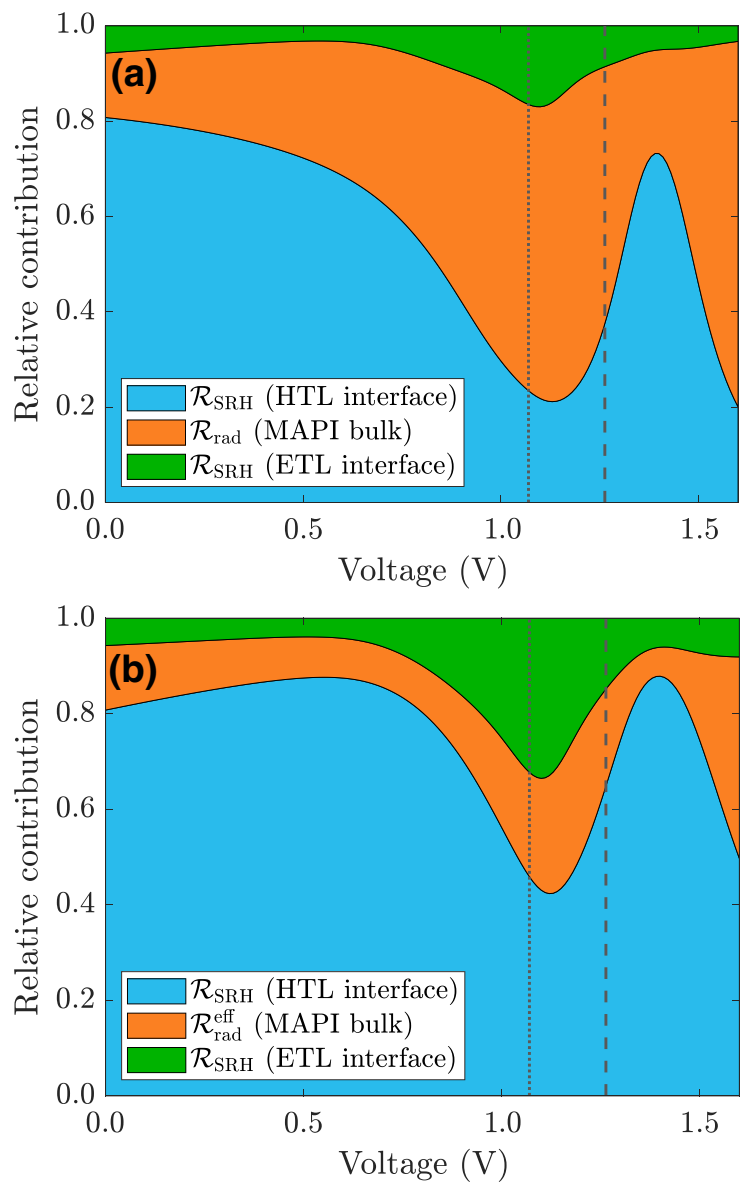

FIG. 5. Relative recombination currents as a function of external voltage for device $B . V_{\mathrm{MPP}}=1.07 \mathrm{~V}$ and $V_{\mathrm{OC}}=1.263 \mathrm{~V}$ are marked with the dotted and dashed lines, respectively. (a) Layer-resolved relative contribution of recombination currents. Radiative recombination in the interface layers and SRH recombination in the bulk are negligible. (b) By considering the effective radiative recombination rate as defined in Eq. (9) the relative contribution of radiative recombination decreases. This decrease is a direct consequence of PR.

the generated charges due to internal reabsorption, while the quantity of Eq. (11) additionally takes into account incomplete reabsorption of the internal emission, similar to the usual internal and external quantum efficiencies defined for incident illumination.

The two efficiencies are plotted in Figs. 6(a) and 6(b), respectively, for both devices around $V_{\mathrm{OC}}$ in the case of $\mathrm{SRH}$ and in the radiative limit. For $V \ll V_{\mathrm{OC}}$ the IQE goes to 1 in every case, i.e., perfect collection of internally generated carriers is observed. Close to $V_{\mathrm{OC}}$ the efficiency drops sharply and finally vanishes for $V \gg V_{\mathrm{OC}}$ regardless of the recombination type considered. This means that for low voltages, diffusion of such carriers is still sufficient for them to be extracted and PR has a measurable impact on the net current at the contacts, while for large forward bias diffusion of internally generated carriers is 

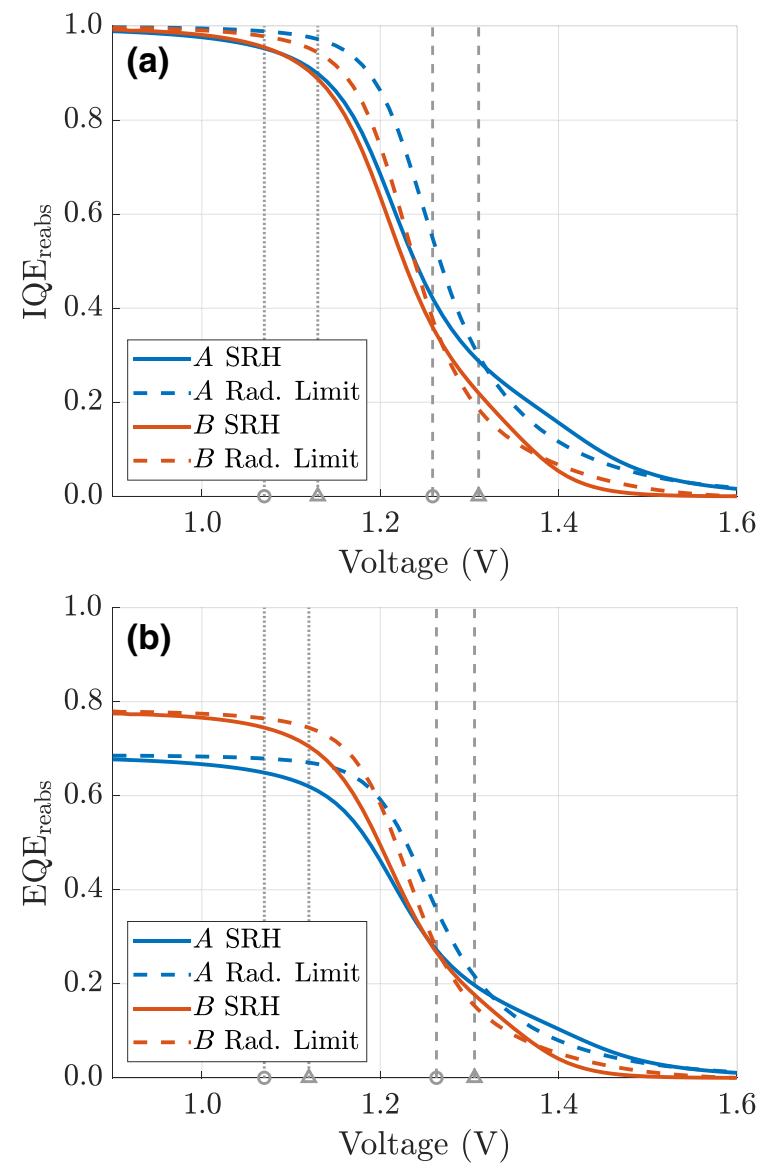

FIG. 6. Quantum efficiencies for reabsorption in both devices in different regimes of charge-carrier recombination. (a) Internal quantum efficiency of reabsorption as defined in Eq. (10). Marked are $V_{\text {MPP }}$ (dotted) and $V_{\mathrm{OC}}$ (dashed) with SRH (circle) and in the radiative limit (triangle) of device $A$. (b) External quantum efficiency of reabsorption as defined in Eq. (11). Marked are $V_{\text {MPP }}$ (dotted) and $V_{\mathrm{OC}}$ (dashed) with SRH (circle) and in the radiative limit (triangle) of device $B$.

fully suppressed and they are confined inside the absorber until they eventually recombine either nonradiatively or radiatively with photon emission into a lossy mode (outcoupled, parasitically absorbed, ...). At this point, there is no measurable impact of PR at the contacts anymore. This can be directly visualized by plotting the dark currents in the radiative limit with and without PR, as shown in Fig. 7. While at low bias the injection current with PR lies considerably below the injection current without PR, they become equal at higher voltages even though the gross radiative recombination current with reabsorption, given by

$$
J^{\mathrm{rad}}(V)=q \int d z \mathcal{R}_{\mathrm{rad}}(z, V)
$$

stays much larger, with the difference in currents being dissipated from the system. At the maximum power point,

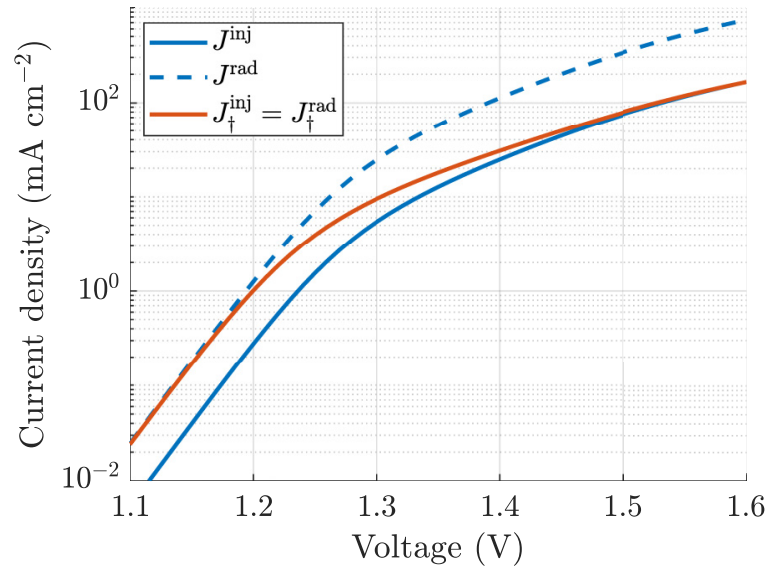

FIG. 7. Dark current densities of device $B$ with and without reabsorption. In the case with reabsorption, the injected current density $\left(J^{\text {inj }}\right)$ lies below the radiative recombination current density $\left(J^{\mathrm{rad}}\right)$ as only a fraction of the latter is actually lost from the system. In the case without reabsorption, the injected current density $\left(J_{\dagger}^{\text {inj }}\right)$ equals the radiative recombination current density $\left(J_{\dot{\dagger}}^{\mathrm{rad}}\right)$.

however, the majority of charge carriers generated through reabsorption still contribute to the terminal current. It is interesting to note that these quantum efficiencies are not necessarily always higher for lower nonradiative recombination, as for both devices the quantum efficiencies in the radiative limit temporarily fall short compared to the SRH case. In both devices this, however, happens for voltages larger than $V_{\mathrm{OC}}$.

On the other hand, the $\mathrm{EQE}_{\text {reabs }}$ shown in Fig. 6(b) follows the same functional dependence as the $\mathrm{IQE}_{\text {reabs }}$, reduced by the fraction of internally emitted radiation, which is not reabsorbed in the system. This fraction that is lost is slightly higher for device $A$ due to the thinner absorber thickness.

To increase the impact of PR, this drop in reabsorption quantum efficiency should be shifted to higher voltages if possible. This can be achieved by increasing the builtin field of the device to counteract the applied forward bias, for example, by doping the charge-transport layers. In Fig. 8 the $\mathrm{IQE}_{\text {reabs }}$ is shown for both devices for increasing doping density ( $n$ doping in PCBM and $p$ doping in PTAA). In both devices the loss in $\mathrm{IQE}_{\text {reabs }}$ is shifted to higher applied voltages (by approximately $130 \mathrm{mV}$ for device $A$ and approximately $80 \mathrm{mV}$ for device $B$ ) and therefore PR has a beneficial impact on device current over a larger voltage range, where the impact is stronger on device $A$ due to the thinner absorber thickness (and hence larger built-in field in the absorber). At the same time, the increase in $V_{\mathrm{OC}}$ is from 1.259 to $1.304 \mathrm{~V}$ for device $A$ and from $1.263 \mathrm{~V}$ to $1.288 \mathrm{~V}$ for device $B$ (see also Fig. S9 within the Supplemental Material [35]). 


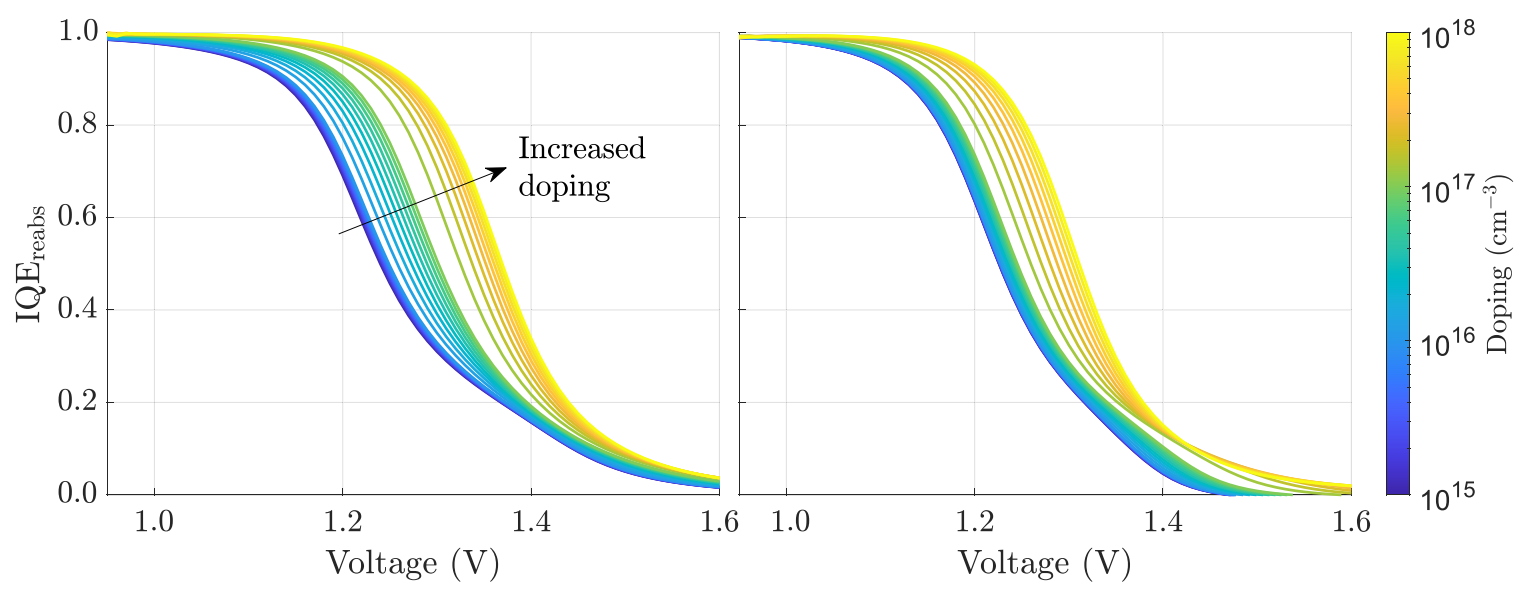

FIG. 8. $\quad \mathrm{IQE}_{\text {reabs }}$ for devices $A$ (left) and $B$ (right) for varying doping densities in the charge-transport layers. The displayed doping values represent donor $(n)$ doping in $\operatorname{PCBM}$ and acceptor $(p)$ doping in PTAA, to equal amounts.

\section{B. Optical-loss analysis}

Aggregate quantities such as the internal and external quantum efficiencies for internally emitted light can be useful to quickly assess the overall efficiency of PR in a device. However, emission and reabsorption possess a strong inherent dependency on the local photonic environment and hence the device geometry. The present modeling approach allows for a detailed assessment of PR efficiency with spatial resolution. It is possible to define an effective radiative rate prefactor in the LED sense as follows:

$$
\begin{aligned}
\mathcal{B}_{\mathrm{rad}}^{\mathrm{eff}, \mathrm{LED}}(z) \equiv & \mathcal{B}_{\mathrm{rad}}(z)-\frac{8}{\pi \hbar^{5} c_{0}^{4} n_{i}^{2}(z)} \\
& \times \int d E_{\gamma} E_{\gamma}^{4} n_{r}\left(z, E_{\gamma}\right) \kappa\left(z, E_{\gamma}\right) \exp \left(-\frac{E_{\gamma}}{k_{B} T}\right)
\end{aligned}
$$

$$
\begin{aligned}
& \times \int d z^{\prime} n_{r}\left(z^{\prime}, E_{\gamma}\right) \kappa\left(z^{\prime}, E_{\gamma}\right) \\
& \times \sum_{\mu, \nu} \int \frac{d^{2} \vec{q}_{\|}}{(2 \pi)^{2}}\left|G_{\mu \nu}\left(\vec{q}_{\|}, z^{\prime}, z, E_{\gamma}\right)\right|^{2} .
\end{aligned}
$$

The second term describes the correction due to reabsorption of radiation emitted at the source point $z$, i.e., $\mathcal{B}_{\text {rad }}^{\text {eff, LED }}$ describes the emitted radiation which is irrecoverably lost from the system. It is now possible to calculate a spatial profile and to reveal possible areas of large optical losses (e.g., plasmon losses close to a metallic electrode), as shown in Fig. 9.

Perfect PR would be theoretically achieved for $\mathcal{B}_{\text {rad }}^{\text {eff, LED }}(z) / \mathcal{B}_{\text {rad }}(z) \rightarrow 0$ everywhere. This, however, would in turn mean that there is zero outcoupling, and due to the reciprocity principle also zero incoupling of external

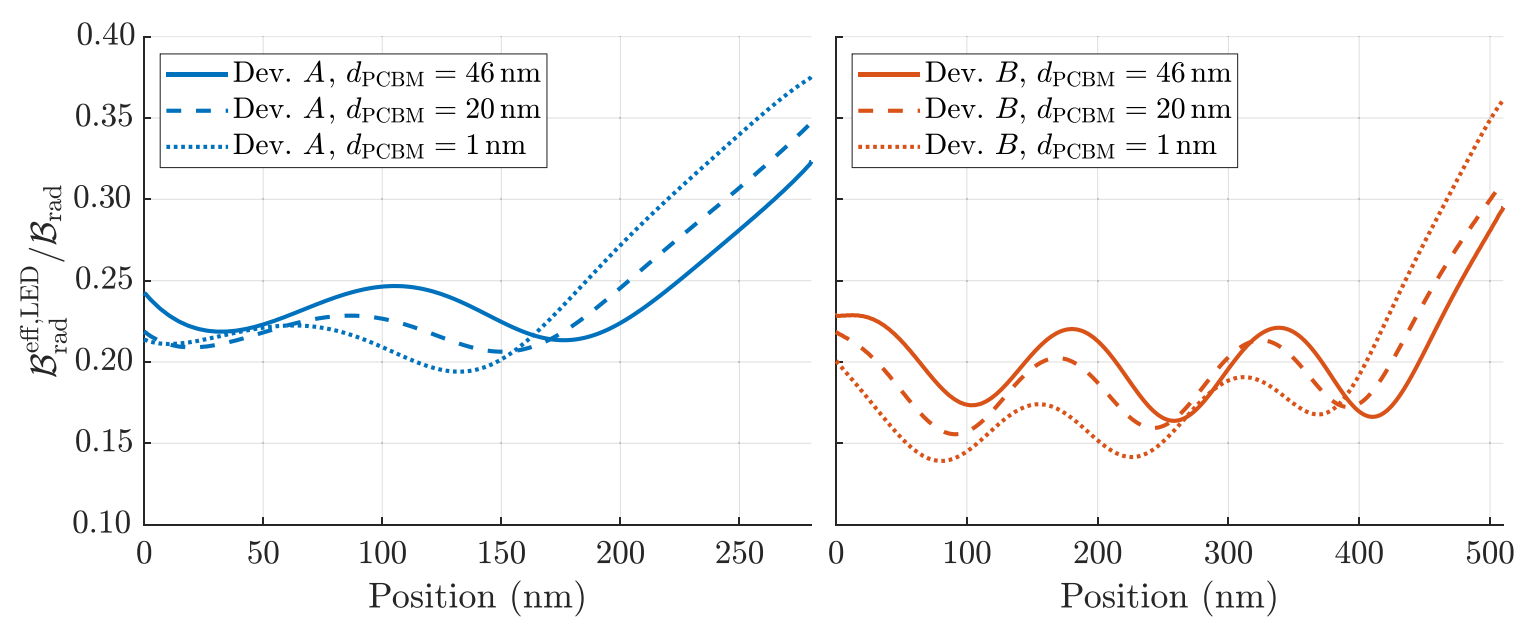

FIG. 9. Plot of the ratio $\mathcal{B}_{\text {rad }}^{\text {eff, LED }}(z) / \mathcal{B}_{\text {rad }}(z)$ inside the MAPI bulk layer for both devices and varying PCBM thickness. The larger the ratio, the more of the locally emitted radiation is not reabsorbed and is lost from the system. 
radiation, i.e., only guided modes exist inside the device. Therefore, for a solar cell, there will always be some degree of internal emission losses. For both devices $A$ and $B$, the loss of internally emitted light increases sharply towards the ETL interface. In general, it is to be expected to have reduced PR efficiency for emission close to the absorber boundaries as a larger portion of light escapes the active absorber. The asymmetry in the present case points, however, to the Ag back reflector as the cause of these losses, which is supported by the data obtained for varying ETL thicknesses. For ETL thicknesses below the original value of $d_{\mathrm{PCBM}}=46 \mathrm{~nm}$, the asymmetry increases and the losses in internally emitted radiation become large, as the active MAPI absorber is moved closer to the back electrode.

For the optical environment of the given devices, the fate of propagating photons emitted into the loss cone is described by the out-of-plane component of the Poynting vector, which is given by [26]

$$
\begin{aligned}
S_{z}\left(z, E_{\gamma}\right)= & \frac{4 E_{\gamma}^{3}}{\hbar^{3} c_{0}^{2} \pi} \int d z^{\prime} n_{r}\left(z^{\prime}, E_{\gamma}\right) \kappa\left(z^{\prime}, E_{\gamma}\right) \\
& \times \exp \left(-\frac{E_{\gamma}}{k_{B} T}\right)\left[\frac{n\left(z^{\prime}\right) p\left(z^{\prime}\right)}{n_{i}^{2}\left(z^{\prime}\right)}-1\right] \\
& \times \operatorname{Im} \int \frac{d^{2} q_{\|}}{(2 \pi)^{2}} \sum_{\mu} \chi_{\mu}\left(\vec{q}_{\|}, z, z^{\prime}, E_{\gamma}\right),
\end{aligned}
$$

with

$$
\begin{aligned}
\chi_{x}\left(\vec{q}_{\|}, z, z^{\prime}, E_{\gamma}\right) \equiv & G_{x x}\left(\vec{q}_{\|}, z, z^{\prime}, E_{\gamma}\right)\left\{\left[\partial_{z} G_{x x}\left(\vec{q}_{\|}, z, z^{\prime}, E_{\gamma}\right)\right]^{*}\right. \\
& \left.+i q_{\|}\left[G_{z x}\left(\vec{q}_{\|}, z, z^{\prime}, E_{\gamma}\right)\right]^{*}\right\}, \quad(15 \mathrm{a}) \\
\chi_{y}\left(\vec{q}_{\|}, z, z^{\prime}, E_{\gamma}\right) \equiv & G_{y y}\left(\vec{q}_{\|}, z, z^{\prime}, E_{\gamma}\right)\left[\partial_{z} G_{y y}\left(\vec{q}_{\|}, z, z^{\prime}, E_{\gamma}\right)\right]^{*}, \\
\chi_{z}\left(\vec{q}_{\|}, z, z^{\prime}, E_{\gamma}\right) \equiv & G_{x z}\left(\vec{q}_{\|}, z, z^{\prime}, E_{\gamma}\right)\left\{\left[\partial_{z} G_{x z}\left(\vec{q}_{\|}, z, z^{\prime}, E_{\gamma}\right)\right]^{*}\right. \\
& \left.+i q_{\|}\left[G_{z z}\left(\vec{q}_{\|}, z, z^{\prime}, E_{\gamma}\right)\right]^{*}\right\} .
\end{aligned}
$$

The resulting photon-flux profile for device $B$ is shown in Fig. 10(a). The strong asymmetry of the photon flux is caused by the Ag back reflector, which can be recognized by the dominant negative photon flux at the left-most and thus top-most interface. While most of the MAPI layer acts as a photon source $\left(d S_{z} / d z>0\right)$, the other layers act as photon sinks $\left(d S_{z} / d z<0\right)$.

By relating the absorption of each layer in the device to the total internally emitted light, a detailed loss analysis of the latter is possible. In order to take into account light emitted into arbitrary modes instead of the loss cone only, the photon flux given by the Poynting vector as described in Eq. (14) is not sufficient and the relative absorptances should be evaluated using the integrated reabsorption rate
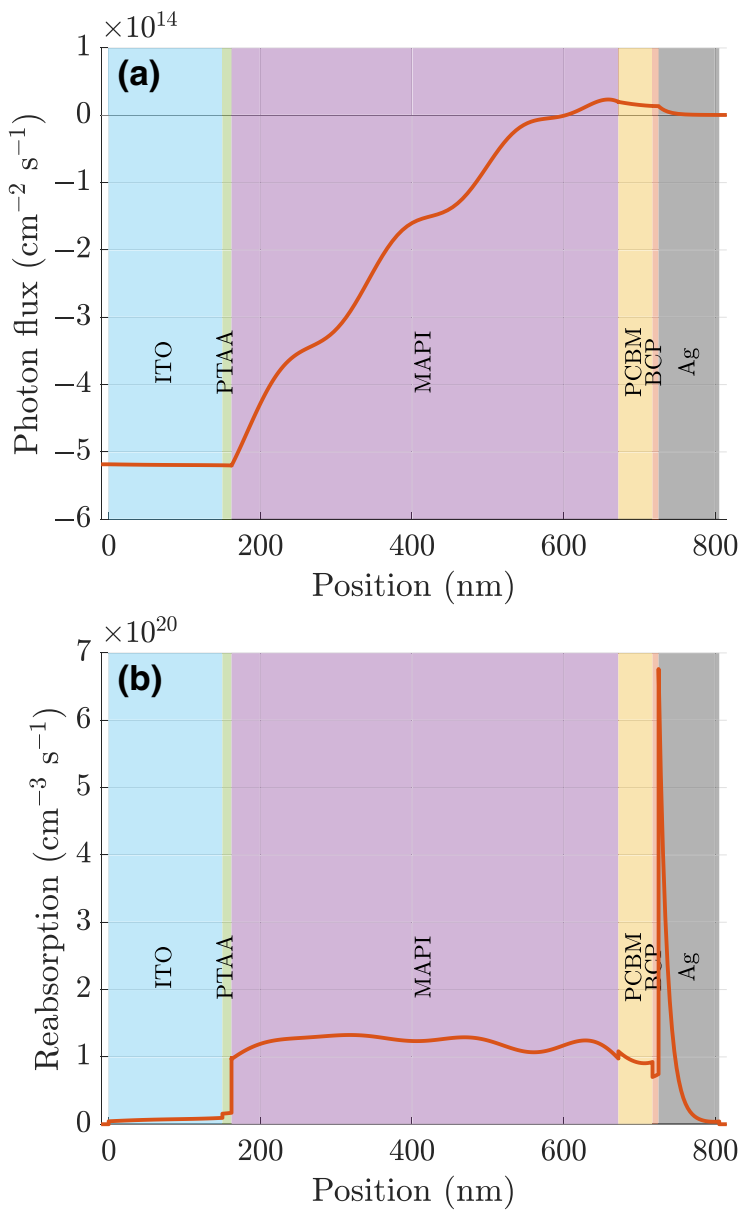

FIG. 10. Photon flux and reabsorption profiles of device $B$ calculated at an external voltage of $V_{\text {MPP }}=1.07 \mathrm{~V}$. (a) Photon flux due to internally emitted radiation. For the sake of reference, the incident photon flux obtained from the integrated solar spectrum amounts to $4.3 \times 10^{17} \mathrm{~cm}^{-2} \mathrm{~s}^{-1}$. (b) Reabsorption rate profile of the internally emitted radiation.

[which is shown in Fig. 10(b) for device $B$ ] as

$$
\tilde{a}_{l}=\left[\int_{\Omega_{l}} d z \mathcal{G}_{\text {reabs }}(z)\right] \times\left[\int d z \mathcal{R}_{\text {rad }}(z)\right]^{-1},
$$

with $l$ the layer index and $\Omega_{l}$ the spatial domain of layer $l$. The resulting absorptances are plotted in Fig. 11(a) for both devices and split into outcoupled and parasitically absorbed contributions, as well as the contribution of reabsorbed radiation in the MAPI layer. The plotted values are calculated at $V_{\mathrm{MPP}}=1.07 \mathrm{~V}$ (same for both devices), as there is only a small variation of the relative contributions over applied voltage due to spatial shifts in radiative recombination (see Figs. S10 and S11 within the Supplemental Material [35] showing the full voltage dependence). The corresponding plots for Fig. 10 for device $A$ are again shown within the Supplemental Material [35]. 

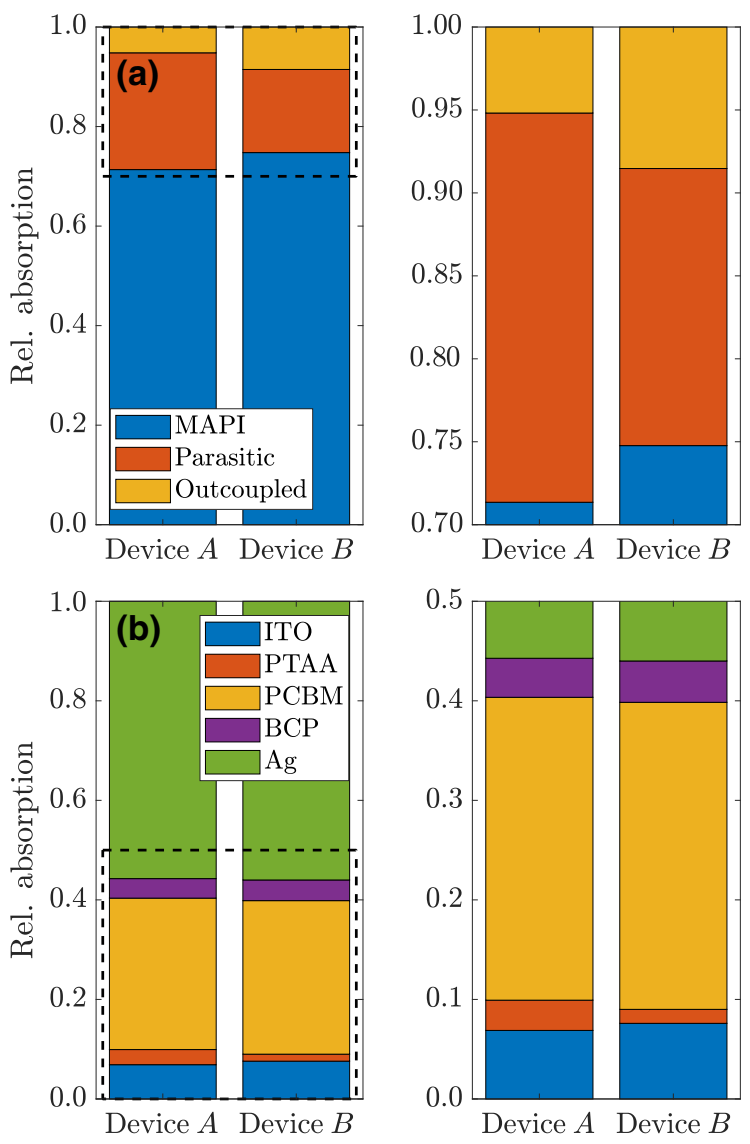

FIG. 11. Loss analysis of the internally emitted radiation calculated at an external voltage of $V_{\mathrm{MPP}}=1.07 \mathrm{~V}$. The two plots in each subplot show the same data with the right one showing an enlarged view of the marked areas to better visualize the differences between devices. (a) Loss channels of the internally emitted radiation, normalized to the total internally emitted power, for both device geometries. (b) More detailed dissection of the parasitic absorption losses with the relative contributions of each layer in the two devices.

From Fig. 10(a) one is led to the conclusion that a large fraction of the radiatively emitted power is outcoupled through the front surface (while outcoupling through the back is negligible as transmission through the $\mathrm{Ag}$ back reflector is small). While this is true for propagating photons in the loss cone, taking all modes into account the outcoupled fraction shrinks to $5.2 \%$ and $8.5 \%$ for device $A$ and $B$, respectively. The largest fraction for both devices is indeed reabsorbed in the MAPI absorber $(71.4 \%$ and $74.8 \%$ ) and is contributing to PR, while parasitic absorption in the other layers amounts to roughly $23.5 \%$ and $16.7 \%$. A more detailed look at the parasitic absorption shown in Fig. 11(b) further supports the conclusions drawn from the analysis of $\mathcal{B}_{\text {rad }}^{\text {eff,LED }}(z) / \mathcal{B}_{\text {rad }}(z)$ : while the top layers (ITO and PTAA) only play a marginal role in parasitic absorption (10\% and $9 \%$ combined, respectively), the largest losses are observed in the Ag back reflector
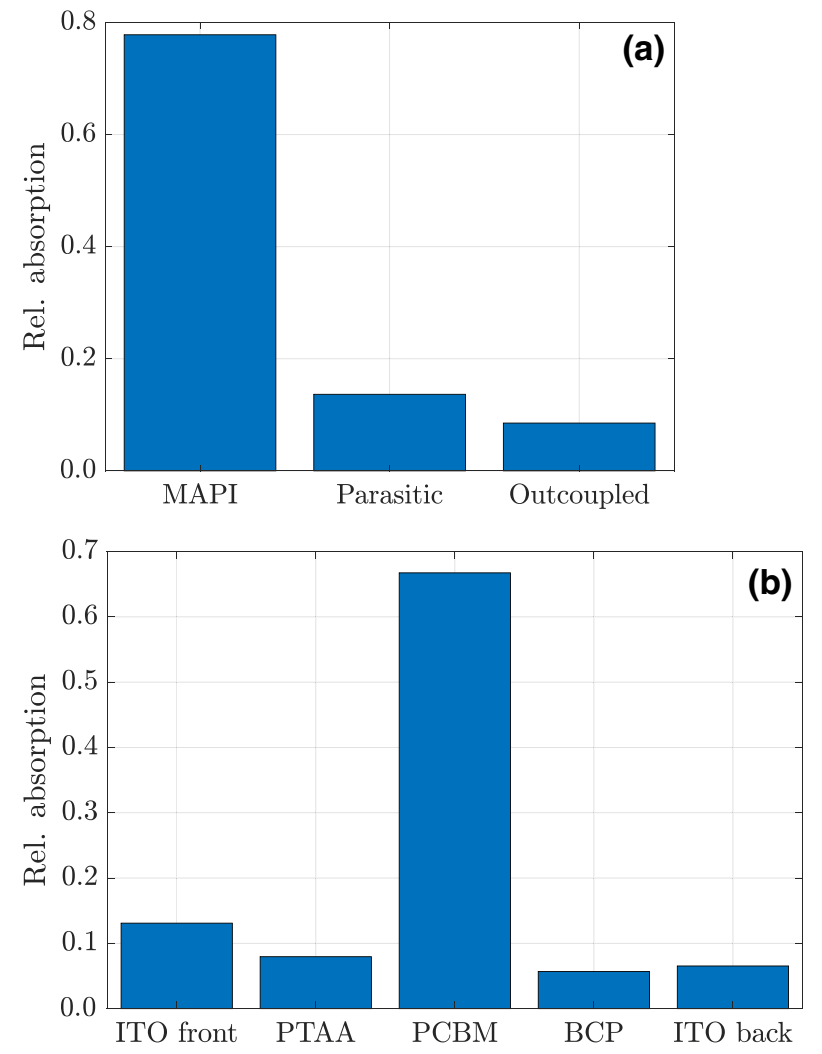

FIG. 12. Optical-loss analysis for internally emitted radiation of device $A$ if the optical properties of the Ag back reflector are replaced by the optical properties of ITO. (a) Loss channels of the internally emitted radiation in device $A$. (b) Dissection of the parasitic absorption in device $A$.

and PCBM ETL (for both devices approximately $56 \%$ and $31 \%$, respectively) which are responsible for the reduced PR efficiency close to the ETL interface inside the MAPI. Yet, it is worthwhile to note that the Ag electrode plays a crucial role in the PR process: without it, large portions of internally emitted light would be lost through the back side of the device and overall the benefits of a back reflector can outweigh the drawbacks when it comes to reabsorption, given the plasmonic losses can be reduced (by e.g., using a semitransparent electrode in conjunction with a Bragg reflector).

To check the influence of the Ag back reflector, we conduct a purely optical analysis using the architecture of device $A$. The optical-loss analysis changes if the back electrode is made transparent, as depicted in Figs. 12 and 13. Keeping everything else the same, the optical properties (complex refractive index) of the Ag back electrode are replaced with the properties of ITO (as is the case in bifacial solar cells). The relative absorptances are plotted in Fig. 12 where the largest share of optical power is absorbed in the MAPI absorber (approximately $78 \%$ ), with a slightly increased share of outcoupled light from $5.2 \%$ to $8.5 \%$. The photon-flux profile is shown in 

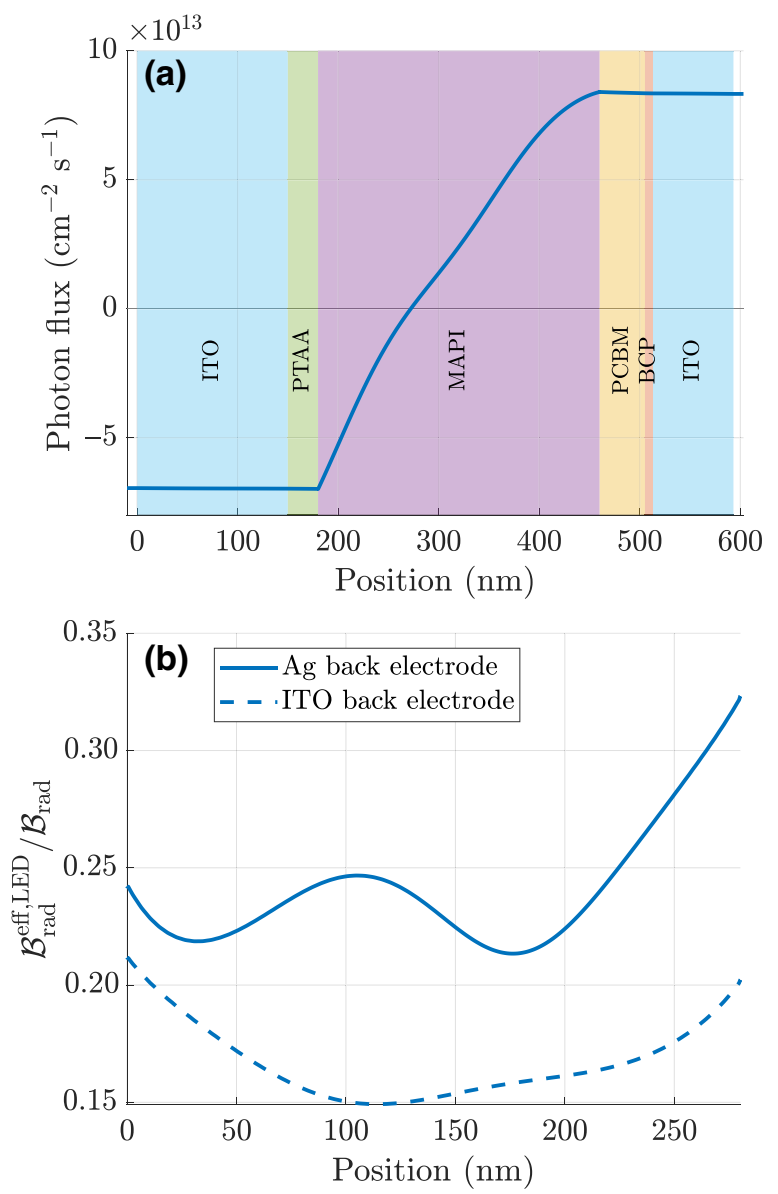

FIG. 13. Photon-flux profile and reabsorption efficiency plot for device $A$ with transparent back electrode. (a) Photon-flux profile of device $A$ evaluated at $V_{\text {MPP }}$ with a transparent back electrode. (b) Comparison of $\mathcal{B}_{\text {rad }}^{\text {eff,LED }} / \mathcal{B}_{\text {rad }}$ of device $A$ with $\mathrm{Ag}$ back electrode and transparent ITO back electrode.

Fig. 13(a), which exhibits a much more pronounced symmetry in comparison with Fig. 10(a), with now increased outcoupling through the back surface.

In Fig. 13(b) the reabsorption efficiency is shown in terms of the ratio $\mathcal{B}_{\text {rad }}^{\text {eff,LED }} / \mathcal{B}_{\text {rad }}$ for the transparent ITO and the reflecting Ag back electrode. Note that even though the losses through outcoupling increased according to the photon flux calculated using the Poynting vector, when taking all modes into account the efficiency of reabsorption is higher (lower value of $\mathcal{B}_{\text {rad }}^{\text {eff,LED }} / \mathcal{B}_{\text {rad }}$ ) in the case of transparent ITO back electrode according to Fig. 13(b), as also confirmed by the increased relative absorptance of the MAPI layer (71.4\% to $78 \%$ ). The plasmonic losses introduced, e.g., by a metallic back reflector present a common problem for PR due to the internal emission into modes with large transverse photon momentum, in contrast to the propagating modes of the external illumination.

\section{CONCLUSION}

A modeling approach is presented that combines an optical model based on a Green's function formalism with a charge-carrier drift-diffusion model for the full optoelectronic simulation of thin-film solar cells where reabsorption of internally emitted light (PR) plays a crucial role. Using the dyadic Green's functions, a detailed-balance compatible expression of the radiative recombination rate prefactor as well as of the internal reabsorption rate as a function of electron and hole densities can be derived. These quantities are then directly considered in the electronic simulation to reach a self-consistent solution of the optoelectronic device equations. Two realistic MAPI single-junction device structures are used as model system by fitting the measured $J-V$ characteristics using the aforementioned model.

For both devices, the influence of PR could be quantified in different regimes of nonradiative recombination, where a potential enhancement in $V_{\mathrm{OC}}$ of up to approximately $50 \mathrm{mV}$ can theoretically be achieved. When taking electronic losses into account, however, this enhancement reduces to approximately $40 \mathrm{mV}$ (radiative limit) and approximately $15 \mathrm{mV}$ (including nonradiative SRH recombination). Particularly the SRH recombination at the HTL and ETL interfaces quenches the radiative recombination, which is dominant in the bulk MAPI. It is, however, useful to distinguish between the gross and effective radiative rates, as only the effective rate describes the actual radiative loss at a given point in the device.

Further, the definition of internal and external quantum efficiencies for reabsorbed radiation, similarly to the efficiencies commonly defined for external illumination, allows a quantification of the extent of the contribution such generated charge carriers make to the current at the terminals as a function of the external voltage. It is shown that for voltages close to $V_{\mathrm{OC}}$ the internally generated charges become trapped in the device and do not contribute anymore to the device current as they are lost to nonradiative or lossy radiative modes before extraction.

It is also shown that through the definition of an effective radiative rate prefactor $\mathcal{B}_{\text {rad }}^{\text {eff,LED }}$ the efficiency of PR can be resolved spatially. While in the bulk the reabsorption efficiency is quite high, it decreases towards the interfaces, where the optical environment additionally plays a crucial role, such as metallic layers introducing plasmon and absorption losses. At the same time, metallic reflectors act as useful barriers to reduce losses incurred by light outcoupling through the rear electrode, as can be analyzed directly by considering the photon fluxes calculated using again the dyadic Green's functions.

In conclusion, PR has a sizable effect on device performance in metal halide perovskite solar cells and should be taken into account in the design process. However, the processes involved are complex and strongly couple optical 
and electronic properties. Using the device simulation approach presented here, these aspects can be considered in a comprehensive and quantitative manner.

\section{ACKNOWLEDGMENTS}

The authors thank Christophe Ballif and Quentin Jeangros (EPFL PV-LAB), as well as Balthasar Blülle and Antonio Cabas Vidani (Fluxim AG) for fruitful discussions. Funding through the European Union's HORIZON 2020 Research and Innovation Programme under Grant No. 953187 (“MUSICODE") is gratefully acknowledged.

[1] J. Jeong et al., Pseudo-halide anion engineering for $\alpha$-FAPbI3 perovskite solar cells, Nature 592, 381 (2021).

[2] A. Al-Ashouri et al., Monolithic perovskite/silicon tandem solar cell with $29 \%$ efficiency by enhanced hole extraction, Science 370, 1300 (2020).

[3] I. L. Braly, D. W. deQuilettes, L. M. Pazos-Outón, S. Burke, M. E. Ziffer, D. S. Ginger, and H. W. Hillhouse, Hybrid perovskite films approaching the radiative limit with over $90 \%$ photoluminescence quantum efficiency, Nat. Photonics 12, 355 (2018).

[4] K. Tvingstedt, O. Malinkiewicz, A. Baumann, C. Deibel, H. J. Snaith, V. Dyakonov, and H. J. Bolink, Radiative efficiency of lead iodide based perovskite solar cells, Sci. Rep. 4, 6071 (2014).

[5] W. Tress, Perovskite solar cells on the way to their radiative efficiency limit - insights into a success story of high opencircuit voltage and low recombination, Adv. Energy Mater. 7, 1602358 (2017).

[6] W.-J. Yin, J.-H. Yang, J. Kang, Y. Yan, and S.-H. Wei, Halide perovskite materials for solar cells: A theoretical review, J. Mater. Chem. A 3, 8926 (2015).

[7] L. M. Pazos-Outón, M. Szumilo, R. Lamboll, J. M. Richter, M. Crespo-Quesada, M. Abdi-Jalebi, H. J. Beeson, M. Vrućinić, M. Alsari, H. J. Snaith, B. Ehrler, R. H. Friend, and F. Deschler, Photon recycling in lead iodide perovskite solar cells, Science 351, 1430 (2016).

[8] J. M. Richter, M. Abdi-Jalebi, A. Sadhanala, M. Tabachnyk, J. P. H. Rivett, L. M. Pazos-Outón, K. C. Gödel, M. Price, F. Deschler, and R. H. Friend, Enhancing photoluminescence yields in lead halide perovskites by photon recycling and light out-coupling, Nat. Commun. 7, 13941 (2016).

[9] T. Kirchartz, F. Staub, and U. Rau, Impact of photon recycling on the open-circuit voltage of metal halide perovskite solar cells, ACS Energy Lett. 1, 731 (2016).

[10] C. Cho, B. Zhao, G. D. Tainter, J.-Y. Lee, R. H. Friend, D. Di, F. Deschler, and N. C. Greenham, The role of photon recycling in perovskite light-emitting diodes, Nat. Commun. 11, 611 (2020).

[11] J. E. Parrott, Radiative recombination and photon recycling in photovoltaic solar cells, Sol. Energy Mater. Sol. Cells 30, 221 (1993).

[12] S. M. Durbinand and J. L. Gray, Numerical modeling of photon recycling in solar cells, IEEE Trans. Electron Devices 41, 239 (1994).
[13] A. W. Walker, O. Hohn, D. N. Micha, B. Blasi, A. W. Bett, and $\mathrm{F}$. Dimroth, Impact of photon recycling on GaAs solar cell designs, IEEE J. Photovolt. 5, 1636 (2015).

[14] F. Staub, T. Kirchartz, K. Bittkau, and U. Rau, Manipulating the net radiative recombination rate in lead halide perovskite films by modification of light outcoupling, J. Phys. Chem. Lett. 8, 5084 (2017).

[15] A. Marti, J. L. Balenzategui, and R. F. Reyna, Photon recycling and shockley's diode equation, J. Appl. Phys. 82, 4067 (1997).

[16] M. G. Abebe, A. Abass, G. Gomard, L. Zschiedrich, U. Lemmer, B. S. Richards, C. Rockstuhl, and U. W. Paetzold, Rigorous wave-optical treatment of photon recycling in thermodynamics of photovoltaics: Perovskite thin-film solar cells, Phys. Rev. B 98, 075141 (2018).

[17] S. Nanz, R. Schmager, M. G. Abebe, C. Willig, A. Wickberg, A. Abass, G. Gomard, M. Wegener, U. W. Paetzold, and C. Rockstuhl, Photon recycling in nanopatterned perovskite thin-films for photovoltaic applications, APL Photonics 4, 076104 (2019).

[18] P. Wurfel, The chemical potential of radiation, Ann. Phys. 15, 3967 (1982).

[19] W. van Roosbroeck and W. Shockley, Photon-radiative recombination of electrons and holes in Germanium, Phys. Rev. 94, 1558 (1954).

[20] R. R. Chance, A. Prock, and R. Silbey, Lifetime of an emitting molecule near a partially reflecting surface, J. Chem. Phys. 60, 2744 (1974).

[21] M. S. Tomaš and Z. Lenac, Decay of excited molecules in absorbing planar cavities, Phys. Rev. A 56, 4197 (1997).

[22] K. A. Neyts, Simulation of light emission from thin-film microcavities, J. Opt. Soc. Am. A 15, 962 (1998).

[23] C. Cho, and N. C. Greenham, Computational study of dipole radiation in re-absorbing perovskite semiconductors for optoelectronics, Adv. Sci. 8, 2003559 (2021)

[24] W. Raja, M. de Bastiani, T. G. Allen, E. Aydin, A. Razzaq, A. u. Rehman, E. Ugur, A. Babayigit, A. S. Subbiah, F. H. Isikgor, and S. de Wolf, Photon recycling in perovskite solar cells and its impact on device design, Nanophotonics 10, 2023 (2021).

[25] Z. Cheng and D. M. O'Carroll, Photon recycling in semiconductor thin films and devices, Adv. Sci. 8, 2004076 (2021).

[26] U. Aeberhard, S. Zeder, and B. Ruhstaller, Reconciliation of dipole emission with detailed balance rates for the simulation of luminescence and photon recycling in perovskite solar cells, Opt. Express 29, 14773 (2021).

[27] S. M. Barnett, B. Huttner, and R. Loudon, Spontaneous Emission in Absorbing Dielectric Media, Phys. Rev. Lett. 68, 3698 (1992).

[28] S. Scheel, L. Knöll, and D.-G. Welsch, Spontaneous decay of an excited atom in an absorbing dielectric, Phys. Rev. A 60, 4094 (1999).

[29] T. Søndergaard and B. Tromborg, General theory for spontaneous emission in active dielectric microstructures: Example of a fiber amplifier, Phys. Rev. A 64, 033812 (2001).

[30] U. Aeberhard and U. Rau, A Microscopic Perspective on Photovoltaic Reciprocity in Ultrathin Solar Cells, Phys. Rev. Lett. 118, 1018 (2017). 
[31] M. Stolterfoht, C. M. Wolff, J. A. Márquez, S. Zhang, C. J. Hages, D. Rothhardt, S. Albrecht, P. L. Burn, P. Meredith, T. Unold, and D. Neher, Visualization and suppression of interfacial recombination for high-efficiency large-area pin perovskite solar cells, Nat. Energy 3, 847 (2018).

[32] Z. Liu, L. Krückemeier, B. Krogmeier, B. Klingebiel, J. A. Márquez, S. Levcenko, S. Öz, S. Mathur, U. Rau, T. Unold, and T. Kirchartz, Open-circuit voltages exceeding 1.26 V in planar methylammonium lead iodide perovskite solar cells, ACS Energy Lett. 4, 110 (2019).

[33] Fluxim AG, SETFOS 5.2: Semiconducting Emissive ThinFilm Optics Simulator (2020).

[34] W. Shockley and W. T. Read, Statistics of the recombinations of holes and electrons, Phys. Rev. 87, 835 (1952).

[35] See Supplemental Material at http://link.aps.org/supp lemental/10.1103/PhysRevApplied.17.014023 for electronic and optical parameters used in simulations, additional electrical characteristics of simulated $J-V$ curves, electricalloss analysis for device $A$, radiative recombination and reabsorption profiles, doping dependency of $V_{\mathrm{OC}}$, voltage dependence of absorptances, and photon flux for device $A$.

[36] M. Abbas and N. Tekin, Balanced charge carrier mobilities in bulk heterojunction organic solar cells, Appl. Phys. Lett. 101, 073302 (2012).

[37] A. Biewald, N. Giesbrecht, T. Bein, P. Docampo, A. Hartschuh, and R. Ciesielski, Temperature-dependent ambipolar charge carrier mobility in large-crystal hybrid halide perovskite thin films, ACS Appl. Mater. Interfaces 11, 20838 (2019).

[38] A. S. Chouhan, N. P. Jasti, and S. Avasthi, Effect of interface defect density on performance of perovskite solar cell: Correlation of simulation and experiment, Mater. Lett. 221, 150 (2018).

[39] E. von Hauff, V. Dyakonov, and J. Parisi, Study of field effect mobility in PCBM films and P3HT:PCBM blends, Sol. Energy Mater. Sol. Cells 87, 149 (2005).

[40] J. H. Heo, S. H. Im, J. H. Noh, T. N. Mandal, C.-S. Lim, J. A. Chang, Y. H. Lee, H.-j. Kim, A. Sarkar, M. K. Nazeeruddin, M. Grätzel, and S. I. Seok, Efficient inorganic-organic hybrid heterojunction solar cells containing perovskite compound and polymeric hole conductors, Nat. Photonics 7, 486 (2013).
[41] C. Motta, F. El-Mellouhi, and S. Sanvito, Charge carrier mobility in hybrid halide perovskites, Sci. Rep. 5, 12746 (2015).

[42] Z. Ni, C. Bao, Y. Liu, Q. Jiang, W.-Q. Wu, S. Chen, X. Dai, B. Chen, B. Hartweg, Z. Yu, Z. Holman, and J. Huang, Resolving spatial and energetic distributions of trap states in metal halide perovskite solar cells, Science 367, 1352 (2020).

[43] P. Vivo, J. K. Salunke, and A. Priimagi, Hole-transporting materials for printable perovskite solar cells, Materials (Basel) 10, 1087 (2017).

[44] C. Q. Xia, J. Peng, S. Poncé, J. B. Patel, A. D. Wright, T. W. Crothers, M. Uller Rothmann, J. Borchert, R. L. Milot, H. Kraus, Q. Lin, F. Giustino, L. M. Herz, and M. B. Johnston, Limits to electrical mobility in lead-halide perovskite semiconductors, J. Phys. Chem. Lett. 12, 3607 (2021).

[45] V. S. Gevaerts, L. J. A. Koster, M. M. Wienk, and R. A. J. Janssen, Discriminating between bilayer and bulk heterojunction polymer:fullerene solar cells using the external quantum efficiency, ACS Appl. Mater. Interfaces 3, 3252 (2011).

[46] Z. C. Holman, M. Filipič, A. Descoeudres, S. de Wolf, F. Smole, M. Topič, and C. Ballif, Infrared light management in high-efficiency silicon heterojunction and rearpassivated solar cells, J. Appl. Phys. 113, 013107 (2013).

[47] Z. T. Liu, C. Y. Kwong, C. H. Cheung, A. B. Djurišić, Y. Chan, and P. C. Chui, The characterization of the optical functions of BCP and CBP thin films by spectroscopic ellipsometry, Synth. Met. 150, 159 (2005).

[48] D. W. Lynch and W. R. HUNTER, in Handbook of Optical Constants of Solids (Elsevier, 1985), p. 275.

[49] R. Santbergen, R. Mishima, T. Meguro, M. Hino, H. Uzu, J. Blanker, K. Yamamoto, and M. Zeman, Minimizing optical losses in monolithic perovskite/c-Si tandem solar cells with a flat top cell, Opt. Express 24, A1288 (2016).

[50] F. Staub, H. Hempel, J.-C. Hebig, J. Mock, U. W. Paetzold, U. Rau, T. Unold, and T. Kirchartz, Beyond Bulk Lifetimes: Insights Into Lead Halide Perovskite Films from TimeResolved Photoluminescence, Phys. Rev. Appl. 6, 044017 (2016).

[51] R. Brenes, M. Laitz, J. Jean, D. W. deQuilettes, and V. Bulović, Benefit from Photon Recycling at the MaximumPower Point of State-Of-The-Art Perovskite Solar Cells, Phys. Rev. Appl. 12, 014017 (2019). 